\title{
Autoestima profesional en docentes beneficiarios del Programa de Postítulo de matemáticas en el contexto de la evaluación docente
}

\author{
Professional self-esteem in teacher who participate in Mathematics Teacher Training \\ Programs in the context of teaching evaluation
Autoestima profissional em professores beneficiários do Programa de Pós-título de Matemática no contexto da avaliação docente

\author{
Christian Miranda J. ${ }^{\text {a, }}$ Karin Wilhelm O., Gabriela Martin, \\ Marcelo Arancibia y Sonia Osses
}

aUniversidad de Chile, Santiago, Chile. Correo electrónico: christian.miranda@u.uchile.cl

\begin{abstract}
RESUMEN
Este artículo describe la relación de la evaluación docente y la autoestima profesional de los profesores de primaria evaluados de la IX y XIV regiones de Chile. Se toma como referente conceptual la Evaluación de Programas Stake (1998) y Autoestima Profesional (Miranda, 2006) en relación a los aportes teóricos y metodológicos de la psicología social. Se registran y analizan autoreportes de un grupo de $(n=126)$ profesores perfeccionados en programas de postitulo de mención en matemáticas que laboran en establecimientos educativos públicos. Entre los hallazgos se encuentra el carácter predictor de la autoestima profesional en relación al resultado de la evaluación del desempeño docente, lo cual tiene implicancias en la gestión de los procesos de formación permanente de profesores del país.
\end{abstract}

Palabras clave: autoestima en profesores, evaluación docente, superación profesional.

\begin{abstract}
This article describes the relation between the teaching evaluation and the mathematic primary teachers' professional self-esteem. It has been taken the Evaluation of Stake's Programs (2009) and Miranda's Professional self-esteem (2005) as a conceptual reference in relation to the theoretical and methodological contributions to the social psychology. Self-reports of a group of teachers $(n=126)$ that have participated in belonging to public educational institutions are being registered and analyzed. Among the findings it is possible to perceive the predictor character of the professional self-esteem in relation to the results of the evaluation of the educational performance, which has implications in the management of the permanent training processes of teachers in the country.
\end{abstract}

Key words: teachers' self-esteem, teaching evaluation, teacher training programs.

\section{RESUMO}

Descreve-se a relação da avaliação docente e a autoestima profissional dos professores primário e avaliados da IX e XIV regiões de Chile. Toma-se como referente conceitual a Avaliação de Programas Stake (2009) e Autoestima Profissional (Miranda, 2005) em relação às contribuições teóricas e metodológicas da psicologia social. Registram-se e analisamse relatórios pessoais de um grupo de $(\mathrm{n}=126)$ professores aperfeiçoados em programas de pós-título de menção em matemáticas que trabalham em instituições educativas públicas. Entre os achados encontra-se o caráter de predição da autoestima profissional em relação ao resultado da avaliação do desempenho docente, o qual tem implicações na gestão dos processos de formação permanente de professores do país.

Palavras chave: autoestima profissional de professores, avaliação docente, formação permanente.

Este estudio forma parte de la investigación "Impacto de la formación permanente de profesores en el aprendizaje escolar" (FONDECYT 1101031) financiada por la Comisión Nacional de Investigación Científica y Tecnológica de Chile (CONICYT). 


\section{INTRODUCCIÓN}

En Chile, la Educación General Básica es considerada como la base de las habilidades, destrezas y conocimientos mínimos que le corresponde por derecho a todo ciudadano. Su objetivo es que el educando adquiera una comprensión global del universo y de sí mismo, lo que debe permitir el despliegue inicial de destrezas y potencialidades que harán del individuo una persona íntegra, consciente de sus actos y socialmente responsable. No obstante, el actual contexto de reforma ha generado, junto con la reestructuración curricular, reivindicaciones sectoriales y nuevo marco regulatorio, una serie de diagnósticos de la realidad educativa, que señalan la ineficacia de la enseñanza primaria a la hora del cumplimiento de tales objetivos, constituyéndose en una desventaja competitiva para el país (OCDE, 2009).

El reconocimiento de esta situación, ha implicado reabrir el debate sobre la formación de los profesores de primaria. Reconocer las debilidades formativas de los docentes "generalistas" y las exigencias del currículo actual en términos de contenidos, ha significado orientar los esfuerzos de desarrollo profesional continuo hacia la formación de profesores con mención, especialmente en los últimos años de la educación primaria (segundo Ciclo Básico) y en aquellos subsectores de alto impacto en el desarrollo científico-técnico como Matemáticas. Además, la evidencia empírica internacional es consistente en señalar que la calidad de los profesores es un factor clave de la mejora educativa. Finalmente, ha sido necesario reorientar el contenido de formación de las propuestas de aprendizaje profesional, dados los cambios del sistema escolar, al pasar los últimos años del segundo Ciclo Básico a la Educación Media y a las dificultades que tienen los docentes para desempeñarse en estos niveles al carecer de formación especializada, producto de lo cual se produce una rotación dañina de éstos por distintos subsectores y ambos niveles. Al respecto, el análisis del tiempo promedio asignado a la formación en las especialidades en las carreras de Educación Básica en las Universidades que participaron el Programa de Fortalecimiento de la Formación Inicial Docente determinó que es inferior al $20 \%$ del tiempo total de formación, con un rango amplio que va entre un $54 \%$ y un $8 \%$, lo que muestra la ambigüedad en este aspecto. De hecho, la mayoría de los programas no distingue entre aprendizaje de los contenidos de los subsectores del currículo escolar y la metodología para enseñarlos (Avalos, 2002).

Como una forma de responder a las dificultades que enfrenta la escuela, el profesor y los centros de formación, ante la creciente demanda social, científica y tecnológica que se les asigna en el aprendizaje de las futuras generaciones, se torna clave la formación permanente que reciben los docentes. Ahora bien, en particular relativo a uno de los aspectos principales introducidos como eje evaluativo de la educación primaria aparece crítica la necesidad de actualizar a los profesores de matemáticas para orientar el aprendizaje escolar hacia estas nuevas metas.

Es así como los resultados en recientes investigaciones señalan, con mucha fuerza, las debilidades formativas y de seguridad en el manejo del contenido de estos profesores. Un ejemplo de ello, es el estudio de los resultados de la prueba TIMMS de 1998 (Mineduc, 2004), donde se relaciona el bajo logro escolar de los estudiantes chilenos y el bajo nivel de confianza para enseñar la disciplina que manifiestan los docentes. En matemáticas, el $45 \%$ de los estudiantes son formados por maestros que declaran tener poca confianza en su preparación para enseñar la materia. Esto es casi tres veces 
el promedio internacional (Miranda, 2008). De acuerdo a la OCDE (2009), esto es un problema casi estructural que se debe a la inadecuada forma de enfrentar el problema del conocimiento del contenido de las materias a enseñar que tienen los profesores, especialmente aquellos que enseñan en los cursos superiores de la enseñanza básica y la baja proporción de docentes especializados (14\%), lo cual se podría relacionar a la desaceleración de aprendizajes que presentan, por ejemplo, los niños de séptimo grado en Matemáticas (PISA, 2007; MIDE UC, 2009).

Es en este marco que la evaluación de tales docentes adquiere importancia social, tomando parte en los debates sobre la calidad educativa, siendo la resistencia de los docentes y la pertinencia de los instrumentos evaluativos temas centrales de la agenda pública durante los últimos años (CPEIP, 2009). De esto se desprende que la evaluación del desempeño docente es un tema sensible para los maestros de primaria y relevante en las políticas de fortalecimiento de la carrera docente, al estar asociada a incentivos económicos (aquellos evaluados como Destacados y Competentes) o de sanción para los que resultan mal evaluados (Básicos e Incompetentes) a través de un Plan de Superación Profesional y eventualmente, su salida del sistema escolar. Lo anterior no solo permite fundamentar la pertinencia de este estudio sino que también abre espacios de análisis sobre el rol de la evaluación en el desarrollo profesional de los docentes, donde uno de los factores claves es la autoestima.

La autoestima de los docentes constituye un factor relevante en su desarrollo profesional. Por tanto, indagar acerca de la situación de los pedagogos y cómo puede verse afectada por factores inherentes a su quehacer, como es el caso de la evaluación es sin duda un desafío nacional. Así, el principal objetivo de este trabajo es describir la incidencia de la evaluación de desempeño docente sobre la autoestima de los profesores de primaria beneficiarios del programa de postitulo de mención en matemáticas sometidos a este proceso, lo que permite reflexionar acerca de las implicancias de la evaluación y el aprendizaje profesional en el potenciamiento de las prácticas pedagógicas, considerando la autoestima docente un factor relevante en su formación profesional y por extensión, de sus estudiantes (Branden, 1995).

Esta investigación considera la evaluación como un juicio de valor que posibilita una reflexión sistemática sobre el quehacer docente, que atiende al contexto, considera globalmente las situaciones, atiende tanto a lo explícito como lo implícito de su práctica y se rige por principios de validez, participación y ética (Stake, 2009). En este escenario, la evaluación docente es una práctica que compromete una dimensión ética no siempre tenida en cuenta y asumida como tal, ya que ésta implica valorar y tomar decisiones que impactan en la cotidianidad de los profesores. Es por eso que cualquier proceso que implique evaluar, más aun si lo que se evalúa son personas, requiere de un proceso reflexivo que asuma una posición de análisis crítico en torno a las acciones que se realizan conjuntamente con las intenciones que se persiguen. Es decir, es necesario preguntarse ¿qué se pretende?, ¿qué valores están involucrados?, ¿cómo se realiza?, ¿qué efectos tiene?, ¿qué papel asumen los evaluadores?, entre otras.

En síntesis, la relevancia y motivación de esta investigación recae en la importancia de describir la incidencia del resultado de la evaluación docente en la autoestima de los profesores de matemáticas, tomando como base que ésta es un fenómeno múltiple y complejo que tiene repercusiones en el ejercicio profesional en lo técnico (práctica docente) y económico (incentivos y apoyos) asociados a este sector laboral. 


\section{MARCO REFERENCIAL}

\section{II.1. ANTECEDENTES DE LA EVALUACIÓN DOCENTE EN CHILE}

En el actual proceso de reforma chilena se ha puesto el acento en el profesor como sujeto de la transformación (OCDE, 2009). Siendo éste quien utiliza o desestima los medios y los materiales en el contexto de lo que son sus tradiciones y concepciones de enseñanza y aprendizaje. Así, las políticas educativas reformistas se fundamentan, en investigaciones que evidencian la influencia del profesor sobre el educando al incidir en el desarrollo de competencias para la vida tales como la autoestima, debido a que esta se forma en la experiencia del aprendizaje y en el convencimiento que el ser humano puede perfeccionarse durante toda su vida (Sanders y River, 1996; Barber y Mourshed, 2008).

La contingencia del proceso de evaluación docente en Chile hace necesario considerar las posibilidades y limitaciones que puede producir en la apreciación que el docente realiza de su labor. En efecto, para Camacho (2004) la autoestima de una persona se ve condicionada a si misma desde el punto de vista de los grupos en que participa y todo aquello que piensen de uno influirá positiva o negativamente en la personalidad o forma de pensar y por ende, en el proceso de desarrollo de las potencialidades humanas; de la inserción de la persona dentro de la sociedad; y de la valoración que uno tenga de sí mismo dependerá lo que haga en la vida y su participación en ella.

Al mismo tiempo, las aproximaciones éticas y políticas a la evaluación subrayan que un punto de tensión del proceso evaluador es el que atañe al empleo que la administración y la comunidad educativa puedan hacer de los informes o certificados de evaluación y de las implicancias derivadas de ella. Otro ámbito de análisis de este proceso dice relación con el enfoque de rendición de cuentas que está a la base de los actuales procesos educativos, donde el resultado de la evaluación docente puede ser visto como factor clave que informa sobre la calidad de la enseñanza, lo cual no solo condicionaría las políticas sectoriales sino que afectaría la percepción social que se tiene de la calidad educativa general (Hargreaves, 1998; Schulmeyer, 2004; Darling Hammond, 2006).

Una de las particularidades del proceso de evaluación docente en Chile ha sido su legitimidad, al ser producto de acuerdos entre el gremio docente, Municipalidades (sostenedores) y Ministerio de Educación. Para Shulman (2005) la postura de los docentes ante los procesos de innovación y mejoramiento representan un fuerte condicionante, si no determinante, del éxito de cualquier iniciativa en ese sentido. Por lo que la evaluación de desempeño docente, aun siendo estipulada en el Estatuto Docente y apoyada por los actores, constituye un factor crítico para los maestros al ser un agente ajeno a su rutina cotidiana y por requerir tiempo valioso para preparación de los materiales de este proceso.

$\mathrm{Si}$ bien lo anterior es atendible, todavía es muy poco lo que se sabe acerca del impacto que la información producida por los sistemas de evaluación de aprendizaje tiene en sus potenciales usuarios. Aún no se ha construido evidencia empírica acerca del modo en que los resultados son analizados y utilizados en las escuelas; el grado en que las familias y la opinión pública reciben y comprenden la información; el modo en que la misma es empleada como insumo en la toma de decisiones de políticas educativas por parte del Ministerio de Educación; y el grado en que las bases de datos son aprovechados por académicos y centros de investigación para producir conocimiento (Wilhelm, 2008; Wilhelm, Martin y Miranda, 2012). 
De este modo, si el proceso de evaluación docente ha sido diseñado desde un enfoque centrado en el desarrollo profesional de los maestros y orientado hacia el cambio pedagógico a través de una reflexión crítica y un mejoramiento de las prácticas, es significativo preguntarse sobre ¿cómo se relaciona la evaluación de desempeño profesional docente con la autoestima profesional de los profesores, según el nivel obtenido en este proceso?

Para responder a la pregunta planteada, es necesario, establecer los parámetros conceptuales sobre los que este estudio se sustenta, así como operacionalizar su abordaje metodológico desde un ángulo técnico, situaciones que a continuación se detallan.

\section{II.2. AUTOESTIMA PROFESIONAL DOCENTE}

El concepto de autoestima se ha secularizado en el sentido que ya constituye parte del habla popular como un término casual y ordinario, sin embargo muchas investigaciones han reafirmado el valor científico que ésta posee producto de la relevancia en la vida del ser humano, al constituir un elemento clave para el desarrollo óptimo en los niveles socioafectivos, dando paso a una de las áreas más importantes en la configuración personal y profesional de cada persona (Voli, 2005).

Existen múltiples investigaciones realizadas en el campo de la Autoestima. Según Mruk (1998) ya para fines de 1995 se contabilizaban más de 6.780 artículos y 557 libros relativos a la autoestima, especialmente desde el ámbito psicológico y sociológico. De este modo, la autoestima aparece como un instrumento conceptual vital y muy significativo, tanto para la perspectiva psicológica como para la sociología. Se ha entendido, en la mayoría de ellas, como lo que piensan las personas de sí mismas y cómo se evalúan como consecuencia de las condiciones sociales básicas y de una predisposición para las conductas subsiguientes, configurándose como un constructo psicosocial para interpretar la experiencia humana.

Branden (1995) define la autoestima como una experiencia fundamental que lleva la vida a su significatividad y al cumplimiento de sus exigencias; es decir, es la confianza en la capacidad de enfrentar los desafíos básicos de la vida y confianza en el derecho a triunfar y a ser felices. Esto se une al sentimiento de ser respetables, de ser dignos y de tener derecho a afirmar las necesidades y carencias, a sostener principios morales y gozar del fruto de los esfuerzos personales, donde la autoestima estaría relacionada con las experiencias vivenciales relacionadas directamente con las necesidades vitales. Esto tiene implicancias importantes para la aproximación conceptual que se quiere llegar en este estudio.

Así, una autoestima positiva se constituiría como una contribución esencial para el proceso vital, entregando elementos para un desarrollo normal y saludable. Lo contrario, es decir, un nivel de autoestima negativo o bajo, podría conducir al sujeto a una desintegración humana y personal. Lo cual constituiría un factor negativo en el proceso de evaluación ya que el impacto provocado por los malos resultados obtenidos, no promueve una superación por parte de los profesores, sino que más bien les provoca una sensación de fracaso, anulando su motivación para desarrollar cambios de mejora o perfeccionamiento. Más aún, el impacto negativo que pudieran sufrir producto de una mala evaluación podría llevarlos incluso, a someterse a tratamientos psicológicos, psiquiátricos u otros para poder enfrentar las consecuencias de este suceso, tal como ya lo han hecho los profesores que actualmente fueron mal evaluados (Millar, 2006). 
Según Wilhelm (2009), una autoestima positiva depende de dimensiones de sentido tales como: i) Seguridad, formado poniendo límites realistas y fomentando el autorespeto y la responsabilidad; ii) Pertenencia, desarrollada fomentando la aceptación, la relación entre las personas, la incorporación a trabajos colectivos y a la creación de ambientes adecuados y positivos; iii) Propósito, que permite la fijación de metas, a través de la comunicación de las expectativas y el establecimiento de relaciones de confianza; y, iv) Competencia, favorecida con la realización de opciones propias y la toma de decisiones, donde la autoevaluación y el reconocimiento de logro son claves.

En el contexto de esta investigación, el modelo que respondería al anterior esquema de sentido sería el profesor evaluado como Destacado o Competente, a diferencia del que no logra satisfacer las características del modelo, es decir, es evaluado como Básico e Insatisfactorio.

Desde esta base, se afirma que en lo más profundo del ser existe una imagen autocreada, que refleja la idea que el sujeto se ha forjado de quién es como persona y cuán valioso es con respecto a otros. Se corresponda o no con la realidad, esta imagen es el punto de referencia con respecto al mundo que le rodea, es su base para tomar decisiones y la guía para todo lo relacionado con su diario gestionar en la vida.

Esta investigación asume que la evaluación docente es una experiencia que va a marcar positiva o negativamente la autoestima de los profesores/as, y al mismo tiempo la forma de enfrentar el proceso y las consecuencias de este va muy ligado a sus emociones, sensibilidad, manera de enfrentar la vida y grado de valoración propia para asumir riesgos y responsabilidades y enfrentarlos de la mejor forma. Ambas características aportar información relevante para establecer la relación de la evaluación docente con la autoestima de estos maestros. Agregando un tercer nivel de análisis, en el sentido de que la autoestima al manifestarse como cambio personal se explicita de manera concreta en el discurso y en la integración del sujeto, lo cual genera retroalimentación constante entre sujeto y el contexto educativo (Ravela, 2003).

Por lo tanto, este trabajo asume la autoestima como una competencia específica de carácter socio-afectiva expresada en el individuo, a través de un proceso psicológico complejo que involucra, tanto a la percepción, imagen, estima y autoconcepto que tiene éste de sí mismo. En éste, la toma de conciencia de la valía personal se va construyendo y reconstruyendo durante toda la vida, tanto a través de las experiencias vivenciales del sujeto, como de la interacción de éste con los otros sujetos sociales, constituyendo el docente un actor clave en la adquisición del fundamento cultural que sustenta la valía que las personas construyen en su identidad. De este modo, la valoración que el docente tiene de sí mismo y la manera en que conceptualiza el valor que la sociedad otorga a la niñez y la educación son elementos necesarios de considerar en su propio proceso formativo.

En este contexto Riviére (1995) sostiene, desde la psicología social, que es necesario superar la lógica de la transmisión que domina los procesos de formación docente. Para este autor, se debe concebir al aprendiz, como sujeto social, que se construye y reconstruye en el conjunto de relaciones sociales que conforman su mundo particular.

Al respecto, la valoración que a lo largo de la vida profesional se forma el docente ha sido objeto de análisis e investigación por parte de numerosos teóricos de la personalidad y la motivación (Quiroga, 2002).

Este interés no es casual, sino que se fundamenta en la enorme importancia que tiene la autovaloración en la formación psico-social del docente, en el proceso de regulación, 
autorregulación y desarrollo profesional, así como también en el rendimiento académico y formación valórica de los alumnos (Alvarado, 2006; Hunt, 2009).

Desde esta perspectiva, la autoestima y el pensamiento son acciones procesadas, elaboradas e internalizadas por el sujeto a través de su práctica. No existe actividad psíquica desvinculada de la práctica; los procesos y contenidos psíquicos están determinados desde las condiciones concretas de existencia. Es decir, son las experiencias concretas, la acción y la práctica las que determinan la subjetividad (Riviére, 1995)

Al mismo tiempo, las investigaciones han evidenciado que un elemento central en las características problemáticas de los estudiantes tales como el bajo rendimiento, la deserción y abandono escolar es la baja autoestima (Andrade, 2000; Andrade y Miranda, 2001). En tal sentido, Vezub (2009) muestra en sus estudios sobre docentes de primaria que una alta autoestima está asociada con la capacidad del docente para innovar en sus prácticas pedagógicas; una baja autoestima disminuye la potencial capacidad del docente en implementar de manera efectiva tales innovaciones, siendo la autoestima un factor mediador del cambio educativo. Ahí recae la importancia de que los profesores posean una buena autoestima ya que su influencia e implicancia en el quehacer profesional es primordial, principalmente por la responsabilidad que tienen como formadores.

De esta manera, se podría decir que el profesor al aprender se siente más competente en su trabajo profesional, lo que significaría que no sólo adquiere conciencia de su propia valía e importancia, sino que al desarrollarla en forma continua, se convierte en un elemento motivador de su seguridad, satisfacción e identidad social como educador. Tal competencia configura la valoración específica que el profesor tiene de su trabajo, y precisamente eso es lo que denominamos autoestima profesional. Por lo que, es probable pensar, que un trabajo que se realiza con un bajo grado de satisfacción afectiva producirá menos tanto cualitativa como cuantitativamente.

Sin duda, todos los aspectos señalados reafirman la importancia que tiene la autoestima del profesor en relación con distintos aspectos del proceso educativo, constituyéndose en un concepto importante de analizar para cualquier estudio de la realidad educativa. Su influencia, tanto en relación al propio desarrollo socio-afectivo como en la construcción del aprendizaje de los alumnos, ratifican el carácter fundamentalmente social del acto educativo y el papel que cabe a los profesores en la tarea del desarrollo social.

\section{METODOLOGÍA}

Producto de las características teóricas y problema epistemológico del estudio se utiliza una metodología cuantitativa de tipo pre-experimental, exploratoria y de diseño descriptivo. Se busca establecer tendencias, identificar áreas, ambientes, contextos y situaciones de estudio, relaciones potenciales entre variable y dar el "tono" de investigaciones posteriores más elaboradas y rigurosas (Hernández, Fernández y Baptista, 2003). Además, este tipo de diseño posibilita narrar lo que significa un proceso de evaluación para el sujeto de tal valoración a través de un componente clave de su personalidad: la autoestima.

La población de la presente investigación, se constituyó por profesores de primaria perfeccionados en programas de postitulo de mención en matemáticas (entre los años 2007 y 2011) en Universidades de la IX región que se desempeñan en escuelas públicas y que fueron evaluados $(\mathrm{N}=211)$ durante el año 2012 en comunas de la IX y XIV 
región (Chile). De esta se extrae una muestra estadísticamente significativa $(n=126)$ que cumplen los requisitos de voluntariedad, contar con título como pedagogo, con estudios de especialidad y trabajar por más de tres años en el establecimiento educacional.

La estrategia de recolección de datos que se utilizó en esta investigación como instrumento para obtener y analizar la información corresponde al Test de Autoestima Docente de Arzola y Collarte (1992), validado a nivel nacional por Miranda (2004) y local por Millar (2006), que consta de 72 ítems organizados según las tres dimensiones internas: i) Identidad, esto es lo que yo soy como educador; ii) Satisfacción, esto es lo que yo siento de mí mismo como educador; iii) Comportamiento, esto es lo que yo hago como educador. Cada una de estas dimensiones posee 24 ítems, de escalamiento tipo Likert, 12 positivos y 12 negativos, que están redactados en función de cinco escenarios rutinarios del profesor: i) profesor-alumnos, ii) profesor-pares, iii) profesor-autoridades, iv) profesor-apoderados y, v) vocación.

De este modo, el instrumento se aplicó a los maestros, antes de iniciar el proceso de evaluación docente y posteriormente como pos-test después de obtenido el resultado de su evaluación, para así observar la relación entre los constructos.

Las técnicas de análisis utilizadas corresponden a estadística descriptiva tales como Medidas de Tendencia Central (Medias Aritméticas, Desviación Típica), Comparación de Medias (Anova de una Vía), Prueba T y de Contraste. Todo lo anterior, mediante el manejo del Programa SPSS (versión 15.0) para la tabulación, transformación y manipulación de los datos procedentes de la aplicación del instrumental antes señalado (Visauta, 1999).

\section{RESULTADOS}

Todos los integrantes de la muestra se sometieron al Test de Autoestima Docente, con el objetivo de observar la relación entre el proceso de evaluación docente y la autoestima que los maestros manifiestan con respecto a diversos sentimientos y conocimientos específicos de su profesión. Las Tablas 1, 2, 3 y 4 presentan de modo sintético los principales resultados del trabajo.

Tabla 1. Relación de Medias Aritméticas según Evaluación docente y Autoestima

\begin{tabular}{|c|c|c|c|}
\hline CLASIFICACIÓN & Pedia Aritmética & 5,03 & 4,78 \\
\hline $\begin{array}{c}\text { Básico - } \\
\text { Insatisfactorio }\end{array}$ & $\mathrm{n}$ & 61 & 61 \\
\hline & Desviación Estándar &, 43 &, 59 \\
\hline & Media Aritmética & 5,33 & 5,56 \\
\hline Competente & $\mathrm{n}$ & 65 & 65 \\
\hline & Desviación Estándar &, 39532 &, 24465 \\
\hline & Media Aritmética & 5,3589 & 5,2933 \\
\hline & $\mathrm{N}$ & 126 & 126 \\
\hline & Desviación Estándar &, 41 &, 63 \\
\hline
\end{tabular}


En la Tabla 1 se presentan los promedios obtenidos en el pretest, y según el desempeño obtenido (Básico-insatisfactorio y Competente) los promedios del postest, junto a la desviación estándar. Se aprecia que los docentes que obtuvieron un resultado Básicoinsatisfactorio en el Proceso de Evaluación docente, sufrieron una disminución residual en el nivel de Autoestima profesional. Al contrario, los docentes que obtuvieron un nivel Competente en el proceso de Evaluación, experimentaron un incremento parcial en el nivel de Autoestima profesional. No se observaron docentes evaluados en la Categoría Destacado.

A partir de la Tabla 1, se puede afirmar que la evaluación de desempeño docente se relaciona con la autoestima de los profesores de matemáticas sometidos a este proceso, al observarse modificaciones estadísticas en la autoestima de los maestros en las tres categorías encontradas. Existiendo una relación negativa en los profesores que fueron mal evaluados, versus una positiva en aquellos que lograron el nivel Competente. También en este análisis se evidencian diferencias significativas, principalmente en el grupo BásicoInsatisfactorio, en donde baja el promedio de 5,03 a 4,78.

Con el fin de realizar un análisis más exhaustivo de los promedios obtenidos y el nivel de autoestima alcanzado por cada integrante de la muestra, es que se llevó a cabo un análisis intra-sujetos para así evidenciar las variaciones que se produjeron explícitamente en cada profesor antes del proceso de evaluación y después de finalizado y obtenido su desempeño.

Tabla 2. Pruebas de Contrastes Intra-Sujetos

\begin{tabular}{|c|c|c|c|c|c|}
\hline Fuente & $\begin{array}{c}\text { Suma de } \\
\text { cuadrados } \\
\text { (III) }\end{array}$ & gl & $\begin{array}{c}\text { Media } \\
\text { cuadrática }\end{array}$ & F & Significación \\
\hline Evaluación &, 33 & 1 &, 34 & 6,3 &, 018 \\
\hline $\begin{array}{c}\text { Evaluación* } \\
\text { Clasificación }\end{array}$ & 2,2 & 1 & 2,1 & 35,9 &, 001 \\
\hline $\begin{array}{c}\text { Error } \\
\text { (Evaluación) }\end{array}$ & 1,6 & 126 &, 055 & & \\
\hline
\end{tabular}

En la Tabla 2, segunda fila, se evidencia que hay diferencias significativas $(\mathrm{p}=0,001)$ en el promedio de autoestima de cada profesor, antes y después de verse sometidos al proceso de evaluación docente.

Además, tercera fila, se indica el efecto de interacción que se da entre la clasificación, es decir, nivel de desempeño obtenido (Básico-insatisfactorio y Competente), y el antes y después, es decir, el nivel de autoestima que el profesor presentaba antes del proceso y después de obtenido el resultado.

Tabla 3. Prueba T para la igualdad de medias: Prueba de Muestras Independientes

\begin{tabular}{|c|c|c|}
\hline $\mathrm{t}$ & $\mathrm{gl}$ & Significación (bilateral) \\
\hline$-2,48$ & 126 &, 005 \\
\hline
\end{tabular}


Este análisis aporta evidencia respecto que la autoestima actuaría como precedente del nivel de desempeño obtenido en la evaluación docente, y no sólo como consecuencia. Esto se plantea a partir del nivel de autoestima que poseían los profesores de matemáticas antes del proceso, en el sentido de que aquellos con menor nivel de autoestima, coincidentemente, fueron los que obtuvieron un desempeño Básico-Insatisfactorio, mientras que los profesores que presentaron niveles mayores de autoestima, asimismo, obtuvieron un nivel de desempeño Competente. Por lo tanto, se constata que hay diferencias de partida en el nivel de autoestima en los profesores/as de la muestra, al momento de someterse al proceso de evaluación, y que posterior a éste, los resultados cumplieron su pronóstico, es decir, los profesores evaluados como Básicos-Insatisfactorios recienten negativamente en su autoestima tal juicio de valor, mientras que los profesores/as evaluados Competentes obtienen un resultado positivo en su autoestima. Esto es consistente con estudios previos (Wilhelm, Martin y Miranda, 2012) sobre el rol de la autoestima de los docentes que laboran en contextos de alta vulnerabilidad y que se desempeñan en la educación pública en Chile.

Tabla 4. Pruebas de los Efectos Inter-Sujetos

\begin{tabular}{|c|c|c|c|c|c|}
\hline Fuente & $\begin{array}{c}\text { Suma de } \\
\text { cuadrados } \\
\text { (III) }\end{array}$ & gl & Media & F & Significación \\
\hline Intercepción & 1765,9 & 1 & 1765,9 & 5645,4 &, 002 \\
\hline Clasificación & 8,7 & 1 & 8,8 & 29,5 &, 000 \\
\hline Error & 7,4 & 126 &, 3 & & \\
\hline
\end{tabular}

Por último, se llevó a cabo el análisis Inter-Sujetos con el fin de comprobar si la diferencia en el nivel de autoestima es significativa según el nivel de desempeño obtenido en la evaluación docente. En efecto, la Tabla 4, evidencia una diferencia significativa en los promedios de autoestima profesional entre los profesores de matemáticas evaluados como Básico-Insatisfactorio y aquellos evaluados Competentes. Este hallazgo es consistente con estudios previos (Miranda, 2005; Millar, 2006) que permitan potenciar un debate respecto del rol mediador de la autoestima docente en el cambio educativo.

\section{DISCUSIÓN Y CONCLUSIONES GENERALES}

La autoestima se fundamenta en la estimación que se tiene sobre las aptitudes y competencias adquiridas y en la manera que estas se aplican al momento de ejecutar una acción. Significa tener la convicción del buen ejercicio de la mente, en la lucidez al pensar, en los procedimientos a través de los cuales se califica, se opta y se resuelve; es seguridad en la capacidad de hacer y seguridad en las facultades de entendimiento.

La satisfacción que se produce ante la realización de determinados objetivos y la participación en eventos significativos, alude al contentamiento que en ocasiones se experimentan, donde el poder hacer y lo pude hacer legan un legítimo deleite que no tiene 
nada en común con la vanidad. Esta satisfacción brota de una esfera emocional opuesta a la arrogancia, a razón de que se experimenta como una retribución positiva al logro (Camacho, 2004; Millar, 2006). En este sentido, los profesores que tienen poca confianza de sus competencias disciplinares tenderían a evitar perfeccionamientos exigentes y labores profesionales que requieren asumir responsabilidades en áreas de alto impacto como las matemáticas (OCDE, 2009).

Por lo tanto, el nivel de Autoestima que posea un profesor de matemáticas antes de someterse al proceso de Evaluación docente configura un factor sustancial en la forma de enfrentar dicho proceso, de desenvolverse, actuar y sentir, y por ende, puede constituir tanto un precedente del resultado que se obtendrá, como una consecuencia del resultado obtenido (Mineduc, 2010).

A partir de lo anterior, se puede dimensionar el importante papel de la autoestima que posea el docente, principalmente en relación a su valía personal y profesional, en donde el Ser, Saber Convivir, Saber ser, y Saber Hacer, sean potenciados y retroalimentados positivamente (Delors, 1996). De esta manera, si se considera la relación que esto puede tener en los educandos de alta vulnerabilidad (procedentes de pueblos originarios y de zonas rurales), las políticas ligadas (formación permanente-evaluación docente) y el contexto de las regiones consideradas tendría efectos positivos y significativos en la calidad de la enseñanza de las matemáticas. Puesto que, como afirma Andrade (2000), la clave para desarrollar la autoestima en los niños está en cómo se sienten los profesores con respecto a sí mismos. Supuesto que es confirmado por los mismos docentes al afirmar uno de ellos que la buena autoestima de los niños requiere de adultos que también tengan una alta autoestima: profesores y padres (Miranda, 2004). Desde otra dirección, Wilhelm (2009) señala que la importancia del desarrollo de la autoestima en el profesor radica principalmente en la relación de comunicación que se establece entre él y los alumnos, donde la autoimagen que el profesor proyecta afectará y condicionará entonces el crecimiento personal de los alumnos que está formando.

Los resultados del estudio, permiten justificar y valorar el significado que posee la autoestima como fenómeno que se relaciona directamente con el desempeño de los docentes de primaria que se perfeccionan en matemáticas. Una autoestima positiva posibilitaría, tanto para el ejercicio y desarrollo profesional del docente como para sus alumnos un adecuado contexto emocional que facilitaría una educación de mayor calidad humana, especialmente sensible dado el carácter rural que caracteriza a gran parte de la población estudiantil de tales regiones (Millar, 2006). En este sentido, es importante reflexionar sobre el carácter específico de la autoestima que el docente de matemáticas que labora en una zona rural desarrolla en un ambiente de alta vulnerabilidad económica y donde el rol social de la educación se torna clave. Por lo tanto urge llevar a cabo investigaciones que aporten más a esta particularidad, o que delimiten líneas de acción en el fortalecimiento de este contenido curricular en contextos de pobreza.

Resulta imprescindible elevar criterios y estrategias que orienten la acción de los agentes evaluadores para llevar a cabo los futuros procesos de evaluación docente sustentados en base a objetivos pertinentes, flexibles y contextualizados al entorno laboral de los maestros, no estandarizando resultados, insensibles a los contextos particulares y en claro desmedro de aquellos docentes que se desempeñan en colegios deprivados, como es el caso de los estudiados en este trabajo. 
Sin duda, todos los aspectos señalados reafirman la importancia que tiene la autoestima del profesor en relación con distintos aspectos del proceso educativo, constituyéndose en un concepto importante de analizar para cualquier estudio de la realidad educativa. $\mathrm{Su}$ influencia, tanto en relación al propio desarrollo psicosocial como en la construcción del aprendizaje de los alumnos, ratifican el carácter fundamentalmente social del acto educativo y el papel que cabe a los profesores en la tarea del desarrollo social (Riviere, 1995; Quiroga, 2002).

A partir del análisis teórico, de los lineamientos, características y fundamentos del proceso de Evaluación de desempeño profesional docente, se puede observar que la evaluación, en general, continúa asociada al control y la sanción más que al desarrollo profesional y a la mejora de las escuelas (Stake, 2009). Las acciones que se emprenden en este campo, aún son respuestas a la coyuntura más que estrategias en el marco de una propuesta integral para asegurar la calidad del trabajo de los docentes.

Por este motivo es necesario apoyar a los docentes, valorar y reconocer su trabajo mediante un sistema que reconozca su esfuerzo y buen desempeño y que los impulse en su desarrollo profesional, además de generar un sistema que contribuya a fortalecer su protagonismo y corresponsabilidad en los cambios educativos.

En cualquier caso, es claro que si se desea mantener e incrementar la motivación de los profesionales de la educación y mejorar las prácticas pedagógicas con el objetivo de obtener aprendizajes más significativos por parte de los estudiantes, es necesario contar con un sistema que discrimine el buen desempeño docente del que no lo es tanto. Pero, es necesario que dicho sistema esté aceptado por la comunidad educativa, sea técnicamente riguroso y sensible a las particularidades locales (Hunt, 2009).

Por otro lado, este estudio asume como limitación el carácter acotado y local de la muestra de profesores, con lo cual su generabilidad es inadecuada. Además, se cree necesario incluir elementos cualitativos a futuros estudios, en especial cuando se trata del análisis de procesos complejos y dinámicos como la evaluación del desempeño docente.

En síntesis, se trata de una nueva búsqueda de sentido, a la vez de continuidad y ruptura con las prácticas investigativas referidas a calidad docente, con el fin de contribuir al debate sobre el lugar que ocupa el profesor en la sociedad, pero también con el propósito de dar una respuesta al problema de disminuir la brecha de capacidad de los docentes de matemáticas que laboran en contextos de alta vulnerabilidad, para determinar si los programas de postitulo en facultades de Educación generan valor agregado en el aprendizaje escolar respecto a otras estrategias en el actual proceso histórico y educativo. De lo anterior, se desprende que todo estudio referido a la relación entre formación permanente, evaluación y autoestima, es necesario para comprender qué aprenden, cómo se sienten y cómo de desempeñan los maestros de primaria en Chile.

\section{REFERENCIAS BIBLIOGRÁFICAS}

Alvarado. L. (2006). Formación continua de profesores en servicio: formación de formadores. Provisionáis da Educação, vol. III, 75-89.

Andrade, M. (2000). Influencia de las inteligencias múltiples, el rendimiento académico previo y el currículo del hogar sobre la autoestima de los alumnos de II $^{\circ}$ Medio de la comuna de 
Santiago. Tesis de Grado para Doctorado en Ciencias de la Educación. Pontificia Universidad Católica de Chile: Santiago.

Andrade, M. y Miranda, Ch. (2001). "Predicción del rendimiento académico lingüístico y lógico matemático por medio de las variables modificables de las inteligencias múltiples y del Hogar", Boletín de Investigación Educacional, n. 16, 301-315.

Arzola, S.; Collarte.C. (1992). Autoestima y Enseñanza Media. Transferencia Pedagógica y Calidad de Aprendizaje. Proyecto FONDECYT No 704-87. CONICYT: Santiago.

Ávalos, B. (2002): Profesores para Chile: historia de un proyecto. Santiago: Ministerio de Educación.

Barber, M. y Mourshed, M. (2008). Cómo hicieron los sistemas educativos con mejor desempeño del mundo para alcanzar sus objetivos. Santiago: San Marino.

Blanco, R. (2008). Eficacia escolar y factores asociados en América Latina y el Caribe. Salesianos Impresores: Santiago.

Branden, N. (1995). Los seis pilares de la autoestima. Paidós: Barcelona.

Camacho, C. (2004). Autoestima Profesional a partir del Perfeccionamiento Docente: Un estudio comparativo. Barquisimeto: Caracas.

CPEIP (2009). Calidad en el desarrollo profesional. Formación continua de docentes. Santiago: MINEDUC.

Darling-Hammond, L. (2006). El derecho de aprender. Crear buenas escuelas para todos. Barceloina: Ariel.

Delors, J. (1996). La educación encierra un tesoro. Madrid: UNESCO.

Hargreaves, A. (1998). International Handbook of Educational Change. London: Kluwer.

Hernández, R.; Fernández, C.; Baptista, P. (2003). Metodología de la Investigación. Madrid: McGraw-Hill.

Hunt, B. (2009). Efectividad del desempeño docente. Una reseña de la literatura internacional y su relevancia para mejorar la educación en América Latina. Santiago: San Marino.

MIDE UC. (2009). Resultados de la Prueba SEPA. Disponible en: < www.mideuc.cl/7/6/2009

Millar, A. (2006). Autoestima de profesores: el caso de los docentes de la Comuna de Valdivia según contexto rural o urbano. Tesis de Grado de Magister en Educación. Universidad Austral de Chile: Valdivia.

MINEDUC(2004). Estudio explicativo sobre resultados TIMMS-R. Santiago: MINEDUC.

MINEDUC (2010). La Evaluación del Desempeño Docente. Santiago: MINEDUC.

Miranda, C. (2004). Impacto del Programa de Becas en el exterior sobre la Autoestima Profesional, el Pensamiento Crítico y la Innovación en las Prácticas pedagógicas de los docentes beneficiados. Tesis de Grado para Doctor en Ciencias de la Educación. Pontificia Santiago: Universidad Católica de Chile.

Miranda, C.(2005). Aproximación a un modelo evaluativo de la formación permanente del profesorado en chile. Estudios pedagógicos, vol. 31, n. 2, 145-166.

Mruk, C. (1998). Auto-estima: investigación, teoría y práctica. Bilbao: Desclée de Brouwer.

OCDE (2009). Informe del Sistema de educación Superior en Chile. Paris: OCDE.

PISA. (2007): Resultados en España. Madrid: Ministerio de Cultura y Educación.

Quiroga, A. (2002). El sujeto en el proceso de conocimiento: modelos internos o matrices de aprendizaje. Buenos Aires: Niño y Dávila.

Ravela, P. (2003). Los próximos pasos. ¿Cómo avanzar en la evaluación de aprendizajes en América Latina? PREAL, n. 20. En línea; disponible en: http://www.preal.cl/Publicacion.asp.

Riviére, P. (1995). Los Esquemas Mentales. Documento de Trabajo. Santiago de Chile: PIIE.

Sanders W. y Rivers J. (1996). Cumulative and Residual Effects of Teachers on Future Student Academic Achievement. Tennessee: ETS.

Shulman L. (2005). Conocimiento y enseñanza: fundamentos de la nueva reforma. Revista de Currículum y Formación del Profesorado, vol. 9, 1-20. 
Schulmeyer, A. (2004). Estado actual de la Evaluación docente en trece países de América Latina. En Maestros en América Latina: nuevas perspectivas sobre su formación y desempeño. Montevideo: PREAL-BID.

Stake, R. (2009). An approach to the evaluation of instructional programs. En Hamilton D. et al., Beyon the numbers game: A reader in educational Evaluation. London: McMillan.

Vezub, L. (2009). El desarrollo profesional docente centrado en la escuela. Concepciones, políticas y experiencias. En línea; disponible en: http://www.oei.es/noticias/spip.php?article7065 Consultado en Julio de 2010.

Visauta, R. (1999). Paquete Estadístico SPSS para estudios sociales. Madrid: McGraw-Hill.

Voli, C. (2005). La autoestima de los docentes. Buenos Aires: Niño y Dávila.

Wilhelm, K. (2008). Impacto de la evaluación de desempeño profesional docente sobre la autoestima profesional en profesores evaluados en la comuna de Valdivia. Tesis de grado de Magíster en Educación. Universidad Austral de Chile: Valdivia.

Wilhelm, K.; Martin, G.; Miranda, Ch. (2012) Autoestima profesional: competencia mediadora en el marco de la evaluación docente. Revista Latinoamericana de Ciencias Sociales, Niñez y Juventud, vol. 10, n. 1, 1-15. 